\title{
AUTOMOTIVE VEHICLE ENGINE MOUNT BASED ON AN MR SQUEEZE-MODE DAMPER: MODELING AND SIMULATION
}

\author{
Bogdan SAPiński, Jacek SnAmina \\ University of Science and Technology, Department of Process Control, Cracow, Poland \\ e-mail:deep@agh.edu.pl; snamina@agh.edu.pl
}

\begin{abstract}
The study investigates the performance of a semi-active vehicle engine mount incorporating an MR damper working in the squeeze mode (MRSQD), summarising its design, operating principles and key characteristics. The mathematical model of the mount is formulated based on the newly developed MRSQD. Two control algorithms are proposed for MRSQD control. The first algorithm (ALG1) uses the inverse model of the engine-frame system, the other is the sliding mode algorithm (ALG2). The effectiveness of the engine mount system is demonstrated in computer simulation.
\end{abstract}

Keywords: engine mount, vibration reduction, MR damper, control, algorithm

\section{Introduction}

The main source of engine vibrations are unbalanced inertia forces in the assembly of a crankshaft, pistons and connecting rods, as well as forces associated with the combustion process (Jędrzejowski, 1986; Kamiński and Pokorski, 1983). Car body vibrations are mostly attributed to road unevenness. Car body-engine interactions cause the vibrations to be transmitted between these two units. As these sources of vibration cannot be entirely eliminated, minimising the dynamic components of the forces transmitted via engine mounts becomes the major issue.

Elastic vehicle engine mounts were first used in the 1930s, based on rubber components, being small in size and relatively cheap (Yu et al., 2001). In the 1960s, the engine mounts were introduced which used purpose-designed hydraulic elements to stabilise the engine (Flower, 1997; Graf and Shoureshi, 1988). In the years to come, these elements were further modified and upgraded (Singh et al., 1992). They allow control of the mount stiffness and damping parameters in a wide frequency range (Helber et al., 1990). However, parameters that are established at the stage of design have to remain unchanged when the system is in operation.

In the recent years, research efforts have focused on active and semi-active elements to be incorporated in engine mounts (Ivers and Dol, 1991). These elements enable more effective reduction of negative interactions between the engine and the car body. Stiffness and damping parameters can be adapted to the mount operating conditions providing the engine mounts with active or semi-active elements, such as MR dampers (Kim, 2014).

The vehicle engine mount considered in this study is provided with an MR damper operating in the squeeze mode (Sapiński and Krupa, 2013; Sapiński and Gołdasz, 2015; Sapiński, 2015). Its design, operating principles and key characteristics are summarised and the mathematical model is developed incorporating the MRSQD (Snamina and Sapiński, 2014). Two control algorithms are proposed for damper control: the first algorithm (ALG1) uses the inverse model of the engine-frame system, the other is the sliding mode algorithm (ALG2) (Imine et al., 2011). The effectiveness of the ALG1 and ALG2 has been simulated in ideal conditions and during their implementation with a semi-active element. 


\section{MR squeeze-mode damper}

The structure of the former version of the MRSQD was described in the notification of inventive design (Sapiński and Krupa, 2015) and in the works (Sapiński and Gołdasz, 2015; Sapiński, 2015). The present version of the device is characterized by a modified magnetic circuit. The objective of this device improvement was to achieve better characteristics taking into account potential applications of the MRSQD. The structure of the MRSQD with the numeric symbols indicating all key components (1-9) is shown in Fig. 1. The hardware features two concentric
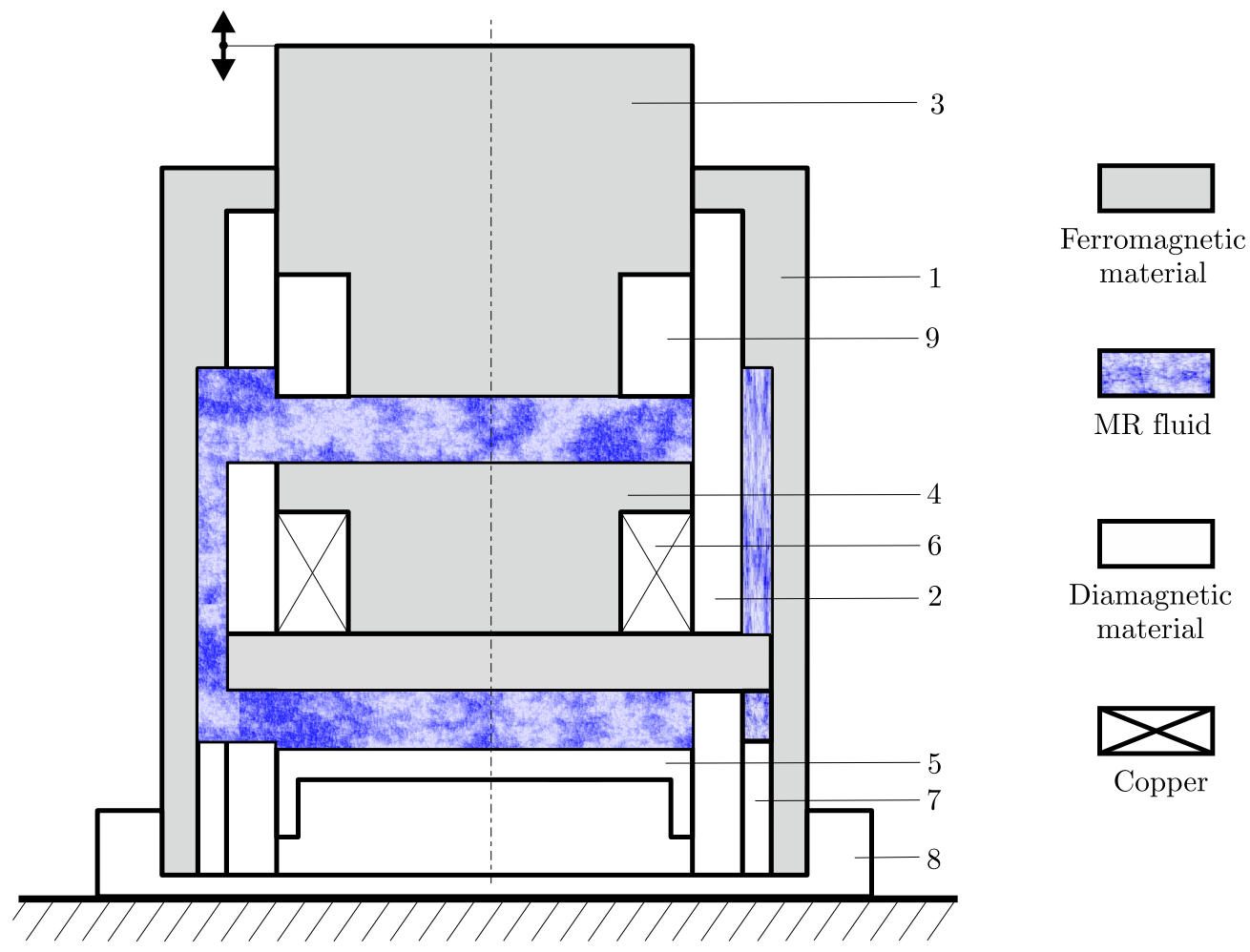

Fig. 1. Structure of the MRSQD

cylinders $(1,2)$. The inner (non-magnetic) cylinder (2) houses the piston (3) with an integrated non-magnetic ring (9), the core assembly (4), and the floating piston (5). The core assembly incorporates the coil (6). The outer cylinder (1) material is ferromagnetic. The distance between the lower surface of the piston and the upper surface of the core is referred to as the control gap of time-variant height $h$. The distance between the piston and the core varies according to the prescribed displacement (force) input. The floating piston below the core assembly separates the MR fluid from the coil spring located in the compensating chamber below the floating piston (5). The chamber incorporates a preloaded coil spring (not revealed in the diagram) for fluid volume compensation. The current in the control coil (6) induces a magnetic field. The magnetic flux generated by the current in the control coil travels through the core and into the control gap, the outer cylinder, and back into the core through radially projected arms in the core base. The inner cylinder of sufficient wall thickness is used to reduce the amount of magnetic flux bypassing the working gap, i.e. magnetic short circuit. All of the components ensure an efficient magnetic flux return path. The flux induced in the control gap upon the application of the coil current effectively modifies the yield stress of the MR fluid and its resistance to flow. As the piston moves downward, the distance between the core and the piston decreases. The excess of the MR fluid is squeezed out of the control gap into the fluid volume between the inner cylinder and the outer housing of the damper, and then into the compensating chamber. The additional 
MR fluid volume that enters the compensating chamber pushes the floating piston against the coil spring. The structure incorporates a non-magnetic ring (7), whereas the base cap (8) is used for fixing the assembly against the ground.

The control coil of the device is represented by the equivalent circuit (see Fig. 2). The circuit consists of constant resistance $R=2.8 \Omega$ and inductance $L(i, h)$ that depends on the applied current $i$ and working gap height $h$. Let us assume that the piston executes sinusoidal motion with a frequency $9 \mathrm{~Hz}$ around to the midpoint of the current gap height with the amplitude $0.7 \mathrm{~mm}$ and recall the relationship $L(i, h)$ determined in (Sapinski and Krupa, 2013). Then, suplying the coil with the step voltage $u=U \cdot 1(t)$ we obtain plots of the current for $U=1.4 \mathrm{~V}$ and $U=2.8 \mathrm{~V}$ and gap height $h=2.16 \mathrm{~mm}$ as shown in Fig. 3. In the steady-state conditions, the constant component of current in the coil is produced by electric input (voltage $u$ ) whilst the variable component is induced by the mechanical input (piston displacement corresponding to the change of the gap height). It can be seen that for the assumed values of $U$, the steady-state current level is $I=0.5 \mathrm{~A}$ and $I=1 \mathrm{~A}$.

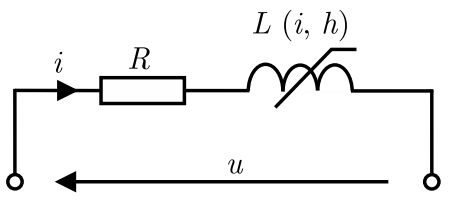

Fig. 2. Equivalent circuit of the control coil

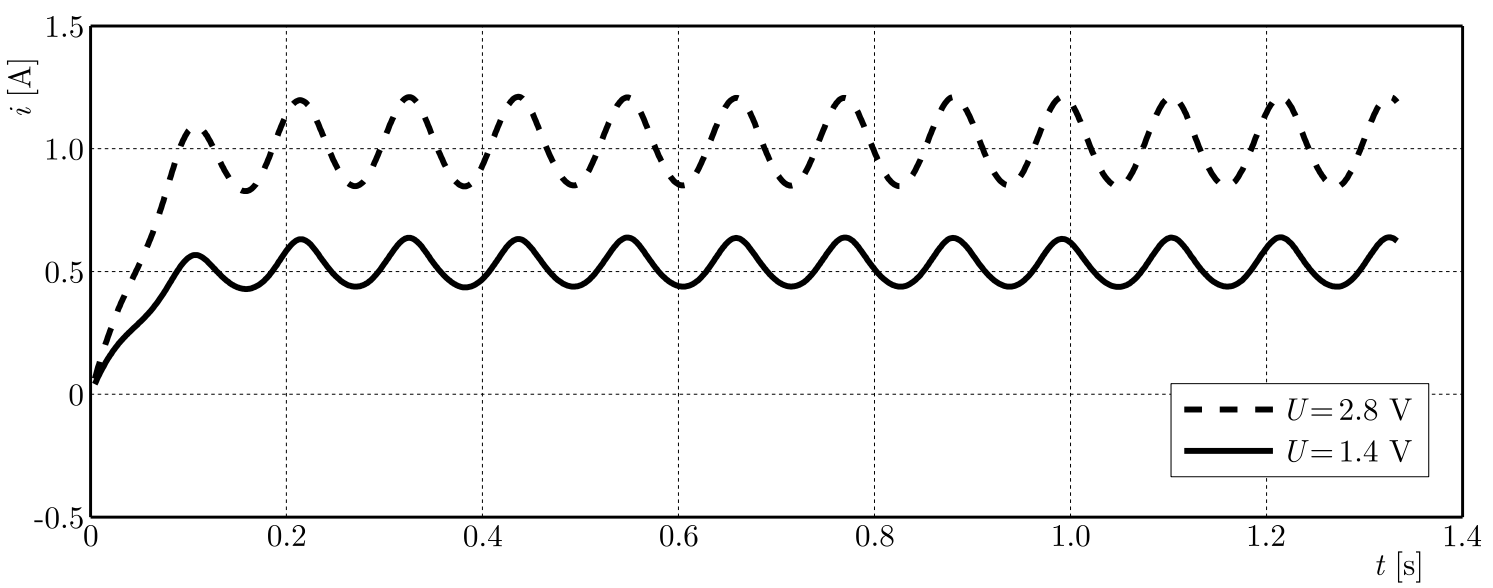

Fig. 3. Current in the control coil at frequency $f=9 \mathrm{~Hz}$

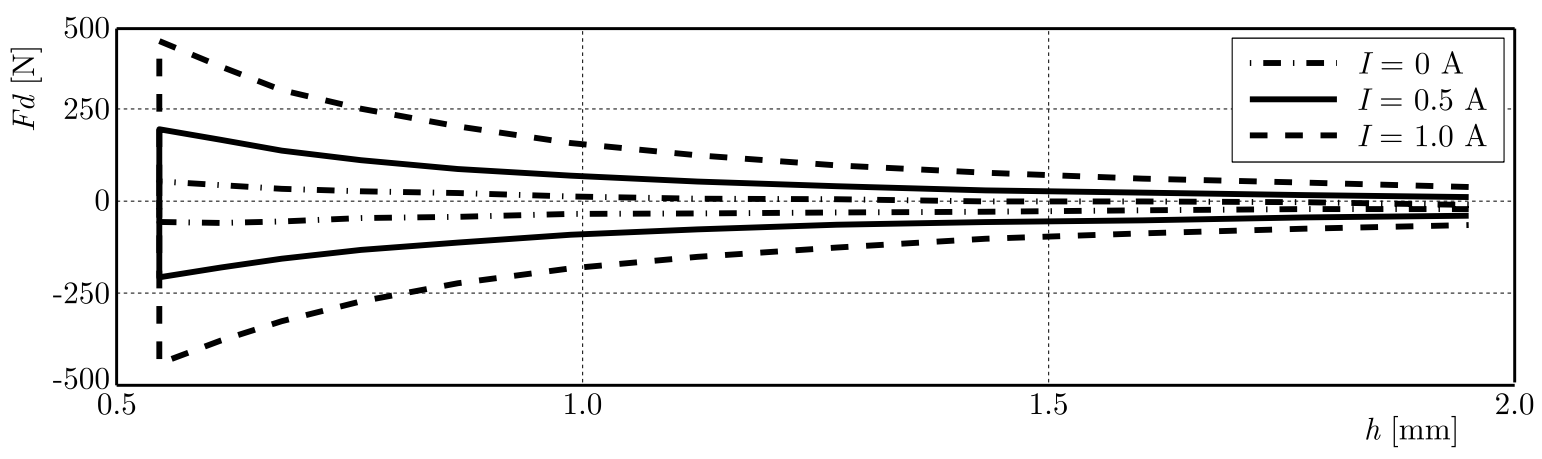

Fig. 4. Force vs. piston displacement for various current levels at frequency $f=9 \mathrm{~Hz}$

The force $F_{d}$ produced by the MRSQD has the following components: force associated with fluid viscosity, inertia force of fluid motion and the force associated with yield stress of the fluid (Sapiński, 2015). In Figs. 4 and 5, we present plots between the force $F_{d}$ and control gap height $h$ and time histories of the force $F_{d}$ for various piston displacement frequencies and for the control 
coil being supplied with no current and the current $I: 0.5 \mathrm{~A}, 1 \mathrm{~A}$. The plots clearly indicate that the applied current in the coil is the major determinant of the damper force whereas for the given current level, the frequency of piston motion (piston velocity) plays a minor role.

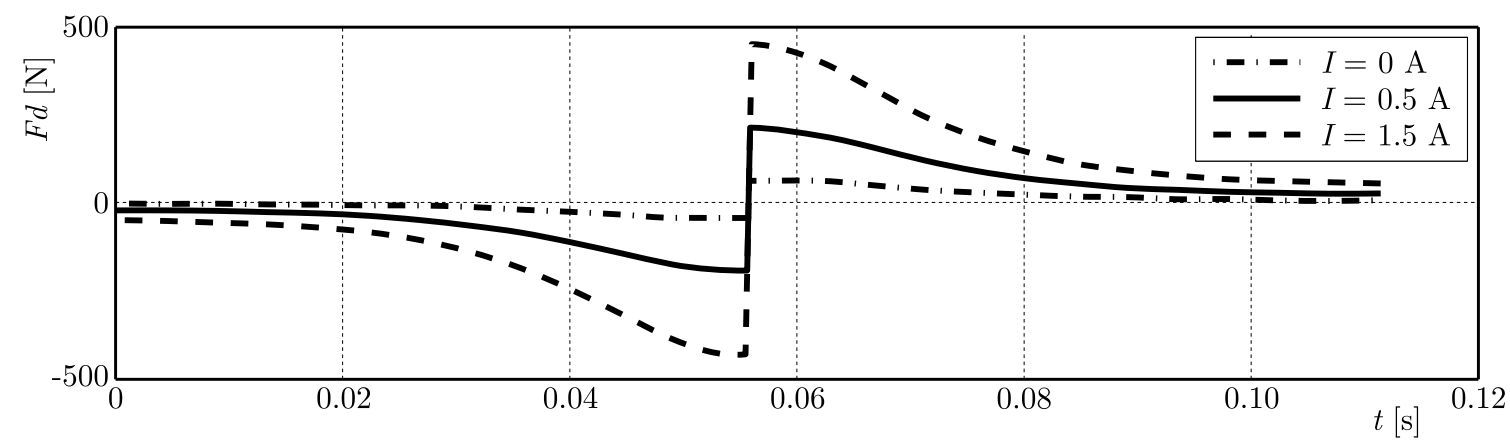

Fig. 5. Time histories of force for various current levels at frequency $f=9 \mathrm{~Hz}$

\section{Modeling of engine mount based on an MR squeeze-mode damper}

Vibrations of the engine and frame linked to the car body are considered as a one process and are investigated using a simplified 2 DOF model schematically shown in Fig. 6 . The engine mount system incorporates the MRSQD. The model embraces this part of the car body which includes the engine.

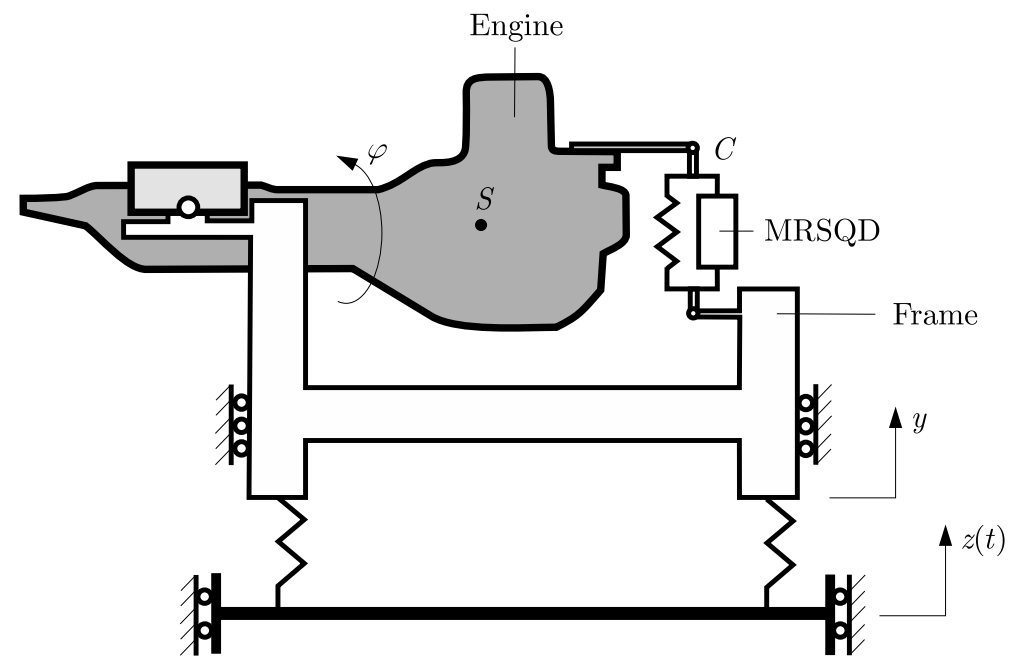

Fig. 6. Schematic diagram of the system

Assuming the kinematic inputs simulating the road unevenness, the following equations are derived

$$
\begin{aligned}
& (M+m) \ddot{y}+m l_{S} \ddot{\phi}+2 b_{p} \dot{y}+2 k_{p} y=2 k_{p} z(t) \\
& J \ddot{\phi}+m l_{S} \ddot{y}+k l^{2} \phi=-F_{d} l
\end{aligned}
$$

where $M$ is the frame mass, $m$ - engine mass, $J$-inertia moment of the engine (incorporating the crankshaft, pistons and rods assembly) with respect to the axis of revolution of the front engine attachment, $F_{d}$ - MRSQD force acting upon the engine block, $l$ - lever arm of the force $F_{d}$ with respect to the axis of revolution, $k$ - stiffness coefficient of a spring connected in parallel to the MRSQD, $l_{S}$ - horizontal distance between the centre of engine mass $S$ and the 
axis of rotation, $\varphi$ - rotation angle of the engine block, $y$-co-ordinate of the frame position, $k_{p}$ - stiffness coefficient of each spring in frame guides, $b_{p}$ - equivalent viscous damping in the frame guides. The co-ordinates $\varphi$ and $y$ describe motion of the system in relation to the static equilibrium position.

The term on the right-hand side of the first equation in system (3.1) is expressed in the physical unit of the force. Designating $F=2 k_{p} z(t)$, we are able to obtain the equivalent diagram of the investigated system (see Fig. 7) in which the kinematic input $z$ is replaced by the applied force input $F$ (preferred in the construction of the laboratory stand).

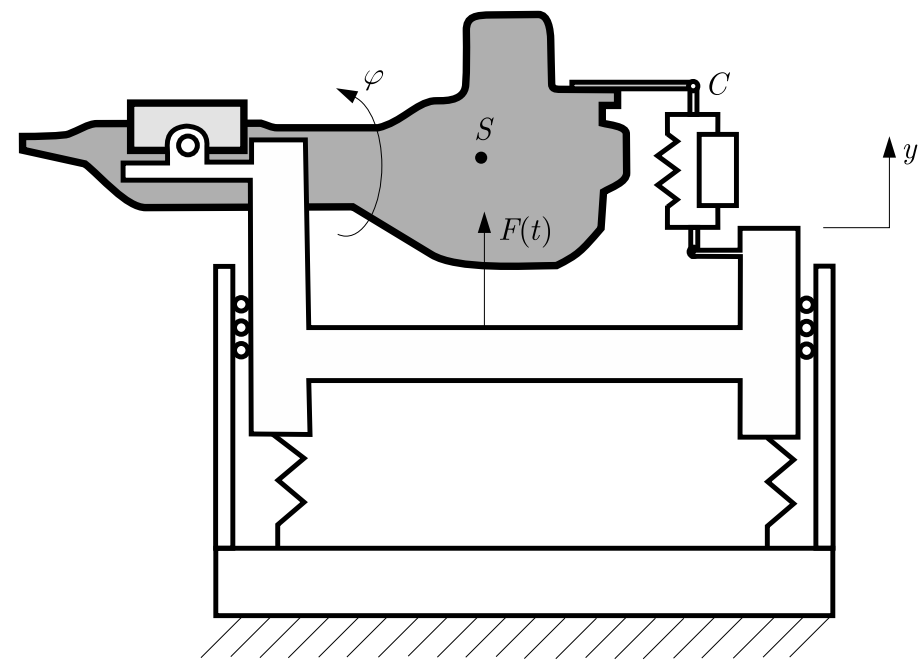

Fig. 7. Modified diagram of the system

The equations governing the system vibrations become

$$
\begin{aligned}
& (M+m) \ddot{y}+m l_{S} \ddot{\phi}+2 b_{p} \dot{y}+2 k_{p} y=F(t) \\
& J \ddot{\phi}+m l_{S} \ddot{y}+k l^{2} \phi=-F_{d} l
\end{aligned}
$$

The MRSQD force acting upon the vibrating object can be approximated with the formula

$$
F_{d}=\beta_{1}\left(\mu, D_{p}\right) \frac{1}{h^{3}} \dot{h}+\beta_{2}\left(D_{p}\right) \tau_{0}(i) \frac{1}{h} \operatorname{sgn}(\dot{h})+\beta_{3}\left(\rho, D_{p}\right) \frac{1}{h} \ddot{h}-\beta_{4}\left(\rho, D_{p}\right) \frac{1}{h^{2}} \dot{h}^{2}
$$

where $D_{p}$ is the piston diameter in the damper, $\mu$ - dynamic viscosity of MR fluid, $\rho$ - density of MR fluid, $\tau_{0}$ - yield stress of MR fluid, $\beta_{1}, \ldots, \beta_{4}$ - coefficients whose values are obtained from measurements.

In the static equilibrium position, the height $h$ of the gap equals $h_{0}$. In this piston position, the co-ordinates $\varphi$ and $y$ are equal to zero. Recalling the MRSQD structure, $h_{0}$ - corresponds to the maximum amplitude of piston displacement with respect to the housing, when the piston is on the same level as the upper surface of the core. Components of the force given by Eq. (3.3) have their physical interpretation: the first one is associated with fluid viscosity, the second one with those properties of MR fluids that are associated with magnetic field induction, and the other two terms are due to MR fluid inertia during the flow between the gap and the compensating chamber. Of major importance is the second term associated with magnetic field induction.

\section{Control algorithms for the MR squeeze-mode damper}

The forces of mount and engine interactions are resultants of static force components compensating for the engine gravity force and dynamic force components associated with the system 
motion. These dynamic forces can be treated as those disturbing the state of equilibrium. The MRSQD force ought to minimise the impacts of forces disturbing the system equilibrium.

The active and semi-active vibration reduction systems use usually sky-hook or LQ algorithms as well as algorithms which employ suitable designed filters. This study presents two special algorithms, designated as ALG1 and ALG2 that allow separation of the sub-system from the rest of the system. Algorithm ALG1 bases on the inverse model of the engine-frame system, and algorithm ALG2 is the sliding mode algorithm.

The main objective of the control in the mount system is to minimise the vibration amplitude of a selected point of the engine. In accordance with algorithm ALG1, the MRSQD interaction force is obtained such as to compensate for the dynamic components of the frame-engine interaction force and to eliminate vibration of the point $C$ of the engine. The algorithm is selected such that the vibrations of the point $C$ should be decaying. The damping decrement expressing the effectiveness of control is dependent on actual parameters of the algorithm. The proposed algorithm can be governed by a force as a function of variables $\varphi$ and $y$ and their derivatives

$$
F_{d}=k y-\left(m \frac{l_{S}}{l}-\frac{J}{l^{2}}\right) \ddot{y}-\beta(\dot{y}+l \dot{\phi})
$$

The frame, engine and the control system with the control algorithm are shown in the block diagram (Fig. 8).

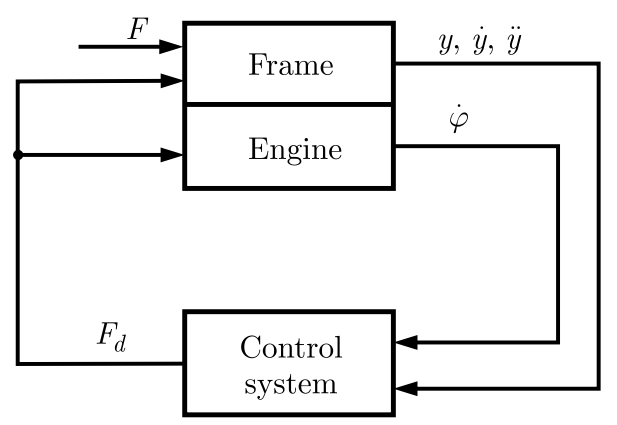

Fig. 8. Block diagram of the investigated system

The coordinates $y$ and $\varphi$ needed to determine the control signal are designated as output signals from the frame and engine blocks. As the damper interacts not only with the engine but with the frame as well, the force $F_{d}$ is given as the input signal to the frame and engine blocks. $F$ is an external force acting upon the frame and disturbing the system equilibrium. Taking into account formula (4.1), in the system of equations (3.2) we obtain equations governing the system motion with feedback

$$
\begin{aligned}
& {\left[M+m\left(1-\frac{l_{S}}{l}\right)\right] \ddot{y}+m \frac{l_{S}}{l} \ddot{w}+2 b_{p} \dot{y}+2 k_{p} y=F(t)} \\
& \frac{J}{l^{2}} \ddot{w}+\beta \dot{w}+k w=0
\end{aligned}
$$

where: $w=y+l \varphi$.

Recalling the inverse model, the force $F_{d}$ is chosen such that the second equation in system (4.2) is not coupleted to the first equation, and that it involves vibration damping. Solution to the second equation determines the solution of the first equation because the force is transmitted onto the frame at the point where the engine is attached.

In accordance with principles of the sliding mode control (Shtessel et al., 2014; Utkin and Chang, 2002), the algorithm contains a sliding variable $\sigma$ being a linear combination of the position and velocity co-ordinates

$$
\sigma=\dot{w}+c w
$$


When the sliding variable is equal to zero $(\sigma=0)$, Eq. (4.3) implicates a sliding surface in a two-dimensional state space. The measure of the distance between the actual trajectory and the sliding surface can be expressed by a function of the sliding variable $\sigma$

$$
V=\frac{1}{2} \sigma^{2}
$$

This is a Lyapunov function. The control is determined basing on the inequality which limits the Lyapunov function derivatives with respect to time, along the trajectory of motion. Assuming that the dynamic component of the force of mount and engine interaction is bounded by $S_{\max }$, the Lyapunov function derivative along the trajectory satisfies the following inequality

$$
\frac{d V}{d t}<\frac{M+m\left(1-\frac{l_{S}}{l}\right)}{(M+m) \frac{J}{l^{2}}-m^{2}\left(\frac{l_{S}}{l}\right)^{2}}|\sigma|\left(S_{\max }-\chi\right)<0
$$

which is the basis for determining the control signal

$$
F_{d}=\chi \operatorname{sgn}(\dot{w}+c w)+\frac{(M+m) \frac{J}{l^{2}}-m^{2}\left(\frac{l_{S}}{l}\right)^{2}}{M+m\left(1-\frac{l_{S}}{l}\right)} c \dot{w}
$$

where the coefficient $\chi$ is given by the formula

$$
\chi=S_{\max }+\frac{(M+m) \frac{J}{l^{2}}-m^{2}\left(\frac{l_{S}}{l}\right)^{2}}{M+m\left(1-\frac{l_{S}}{l}\right)} \frac{\alpha}{\sqrt{2}}
$$

In order to make the control signal derived from Eqs. (4.6) and (4.7) be implemented, it is required that the parameter $S_{\max }[\mathrm{N}]$ should be first determined as it imposes a limit on force disturbing the system equilibrium. Besides, the parameters $c[1 / \mathrm{s}]$ and $\alpha\left[\mathrm{m} / \mathrm{s}^{2}\right]$ should be assumed, expressing the inclination of the sliding surface. The value of the parameter $S_{\max }$ can be estimated through investigating the system vibrations or from measurements.

The investigated vibration reduction system incorporating the MRSQD is a semi-active system. In accordance with the fundamental principle of semi-active systems, it is assumed that the system is capable of reproducing the force implicated by the suggested algorithms as long as the power resulting from damper-object interactions should be negative. When this power is positive, the semi-active system interaction force is equal to zero and the power delivered by the semi-active system will be zero, too. This condition can be written as follows

$$
F_{d(e f)}=\left\{\begin{array}{lll}
F_{d} & \text { if } & F_{d}(\dot{y}-\dot{w})<0 \\
0 & \text { if } & F_{d}(\dot{y}-\dot{w}) \geqslant 0
\end{array}\right.
$$

where $F_{d}$ is the force implicated by the control algorithm, $F_{d(e f)}$ effective semi-active damper force. The relative velocity is the difference between velocity of the point the damper is attached to the engine (point $C$, see Fig. 6) and the velocity of the point where the MRSQD is attached to the frame.

\section{Simulation of engine mount based on MR squeeze-mode damper}

Recalling the mathematical model outlined in Section 4 and using algorithms ALG1 and ALG2, simulations have been performed on the system vibration in the open loop and closed loop 
configuration. Parameters in the simulation procedure were: frame mass $M=60 \mathrm{~kg}$, engine mass $m=80 \mathrm{~kg}$, inertia moment of the engine $J=40 \mathrm{kgm}^{2}$, horizontal distance between the engine centre of gravity and the axis of rotation $l_{S}=0.5 \mathrm{~m}$, distance between the damper attachment point and the axis of rotation $l=0.85 \mathrm{~m}$, stiffness coefficients $k_{p}=6.6 \cdot 10^{3} \mathrm{~N} / \mathrm{m}$ and $k=4 \cdot 10^{4} \mathrm{~N} / \mathrm{m}$, coefficient of equivalent viscous damping $b_{p}=100 \mathrm{Ns} / \mathrm{m}$. A sinusoidal excitation $F(t)=F_{0} \sin (2 \pi f t) ; F_{0}=166 \mathrm{~N}, f=9 \mathrm{~Hz}$ has been assumed.

In the first stage, simulations were performed to determine the following parameters: displacement $w$ of the point $C$ and displacement $y$ of the frame in the open-loop configuration. Simulation results obtained for the current level $I=0.5 \mathrm{~A}$ in the MRSQD control coil are shown in Fig. 9.
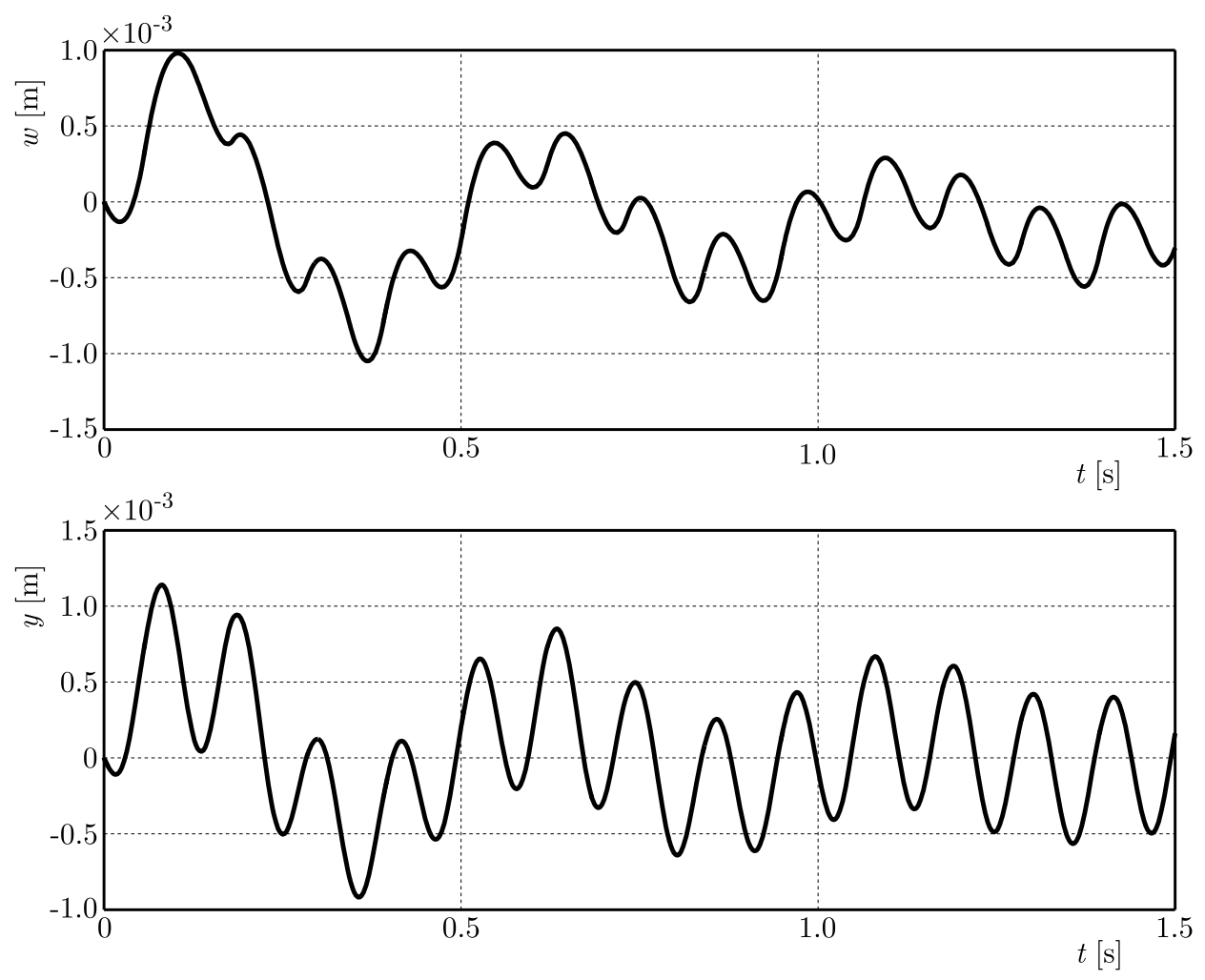

Fig. 9. Time histories of the engine point $C$ and frame displacements (open-loop configuration)

Simulation results obtained using the ideally reproduced control signal in accordance with the algorithm ALG1 are summarized in Fig. 10, the value of the parameter is $\beta=200 \mathrm{Ns} / \mathrm{m}$. In accordance with the algorithm ALG1, the vibration amplitude of point $C$ of the engine decreases and its position asymptotically tends to the static equilibrium position. At the same time the amplitude of frame vibration remains almost unchanged.

Simulation results obtained using the ideally reproduced control signal in accordance with the control algorithm ALG2 are summarised in Fig. 11, the value of the parameters $c=2001 / \mathrm{s}$, $\alpha=10 \mathrm{~m} / \mathrm{s}^{2}, S_{\max }=200 \mathrm{~N}$. When the sliding variable $\sigma$ and the associated sliding surface are introduced, motion of the point $C$ is now governed by a decreasing exponential function corresponding to the movement along the sliding surface. The position of the point $C$ asymptotically tends to the static equilibrium position whilst the amplitude of frame vibration remains almost unchanged.

The assumption made during the second phase of simulations was that the algorithms ALG1 and ALG2 were to be implemented using a semiactive damper. The simulation procedure uses condition (4.8) which yields the effective force value $F_{d(e f)}$. Simulation data are summarised in 

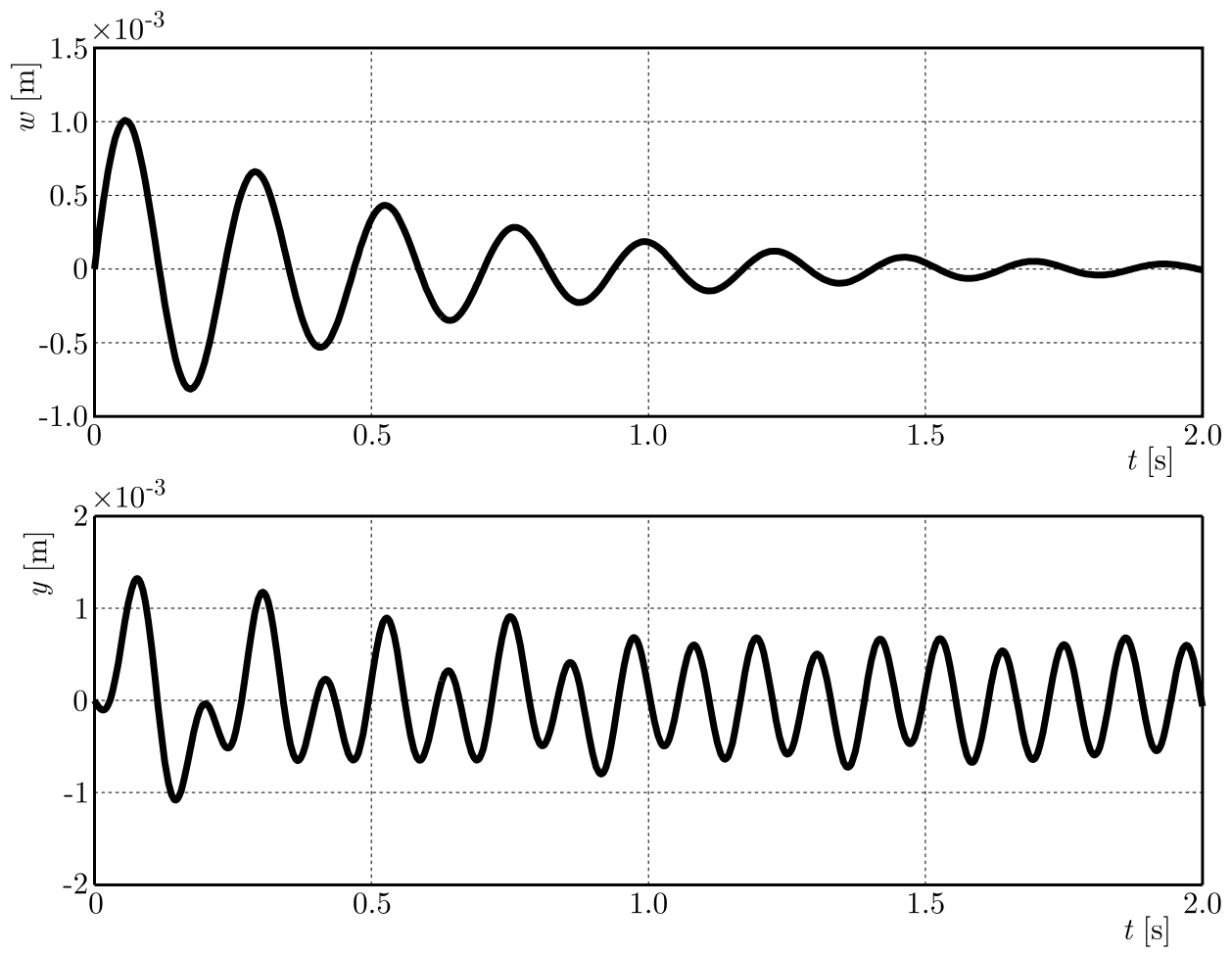

Fig. 10. Time histories of the engine point $C$ and frame displacements (algorithm ALG1)
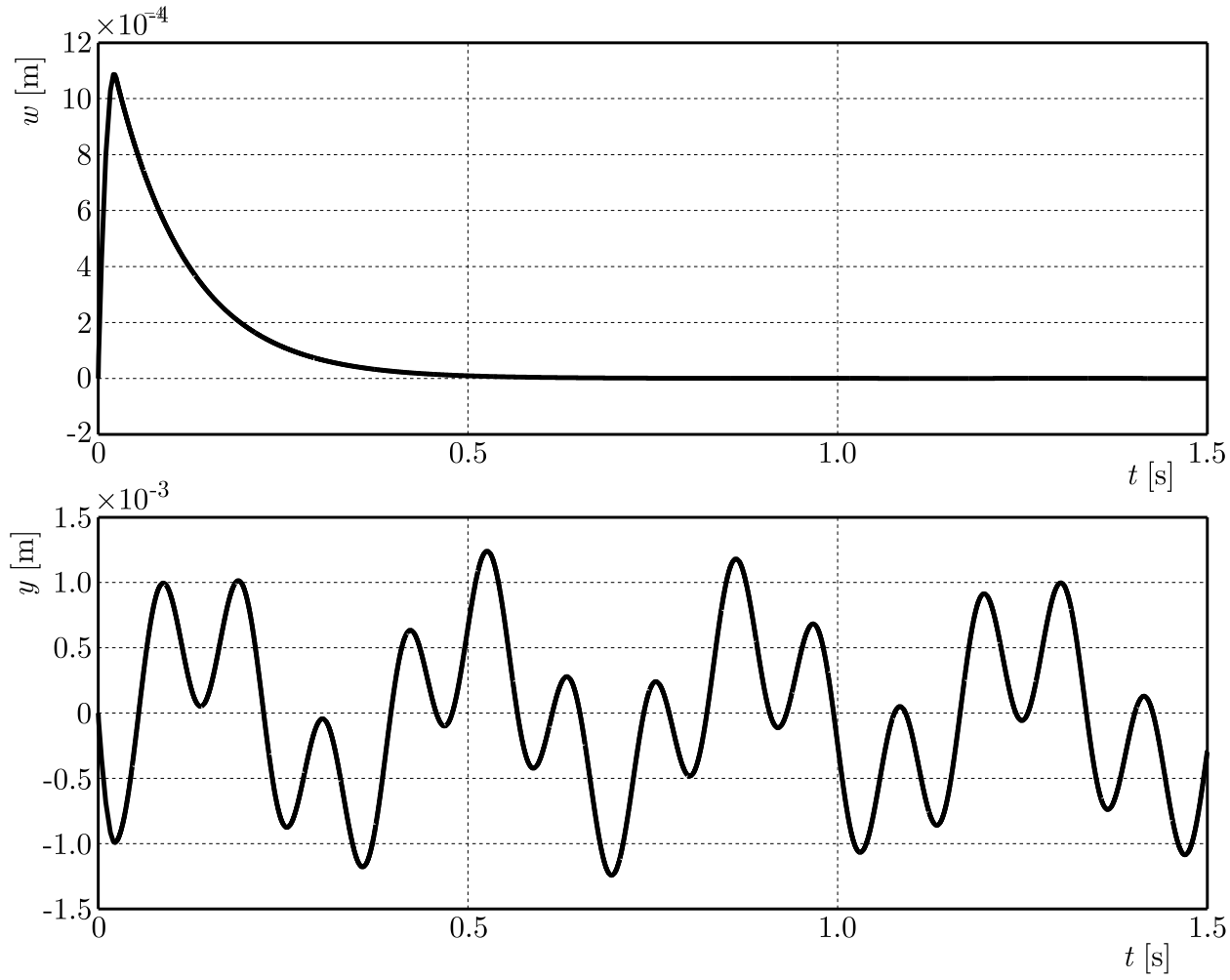

Fig. 11. Time histories of the engine point $C$ and frame displacements (algorithm ALG2)

Figs. 12 and 13, for ALG1 and ALG, respectively. The results show that vibration reduction effectiveness deteriorates in relation to that achievable in the first stage of simulations, particularly in the case of ALG1. 

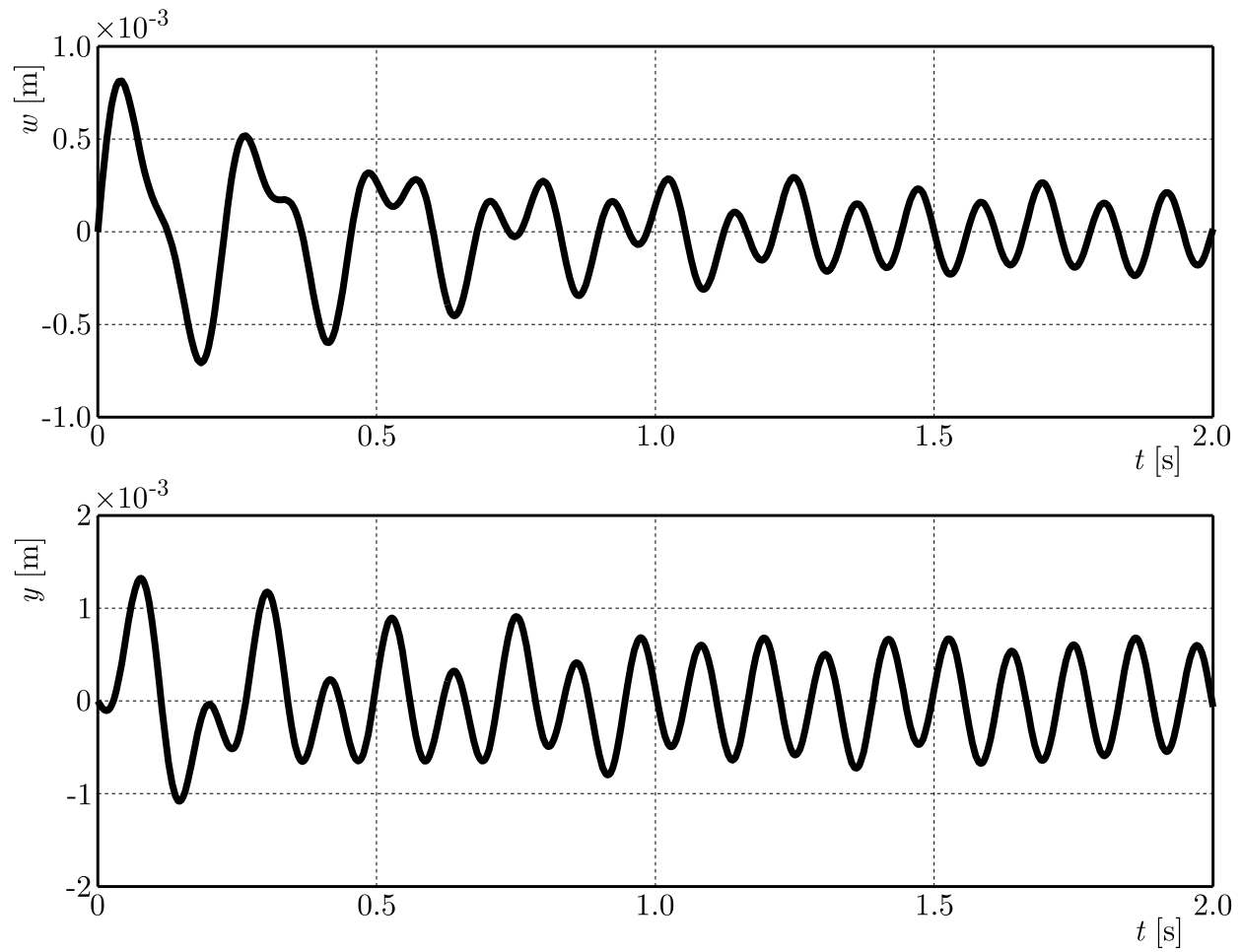

Fig. 12. Time histories of the engine point $C$ and frame displacements (algorithm ALG1, semi-active implementation)
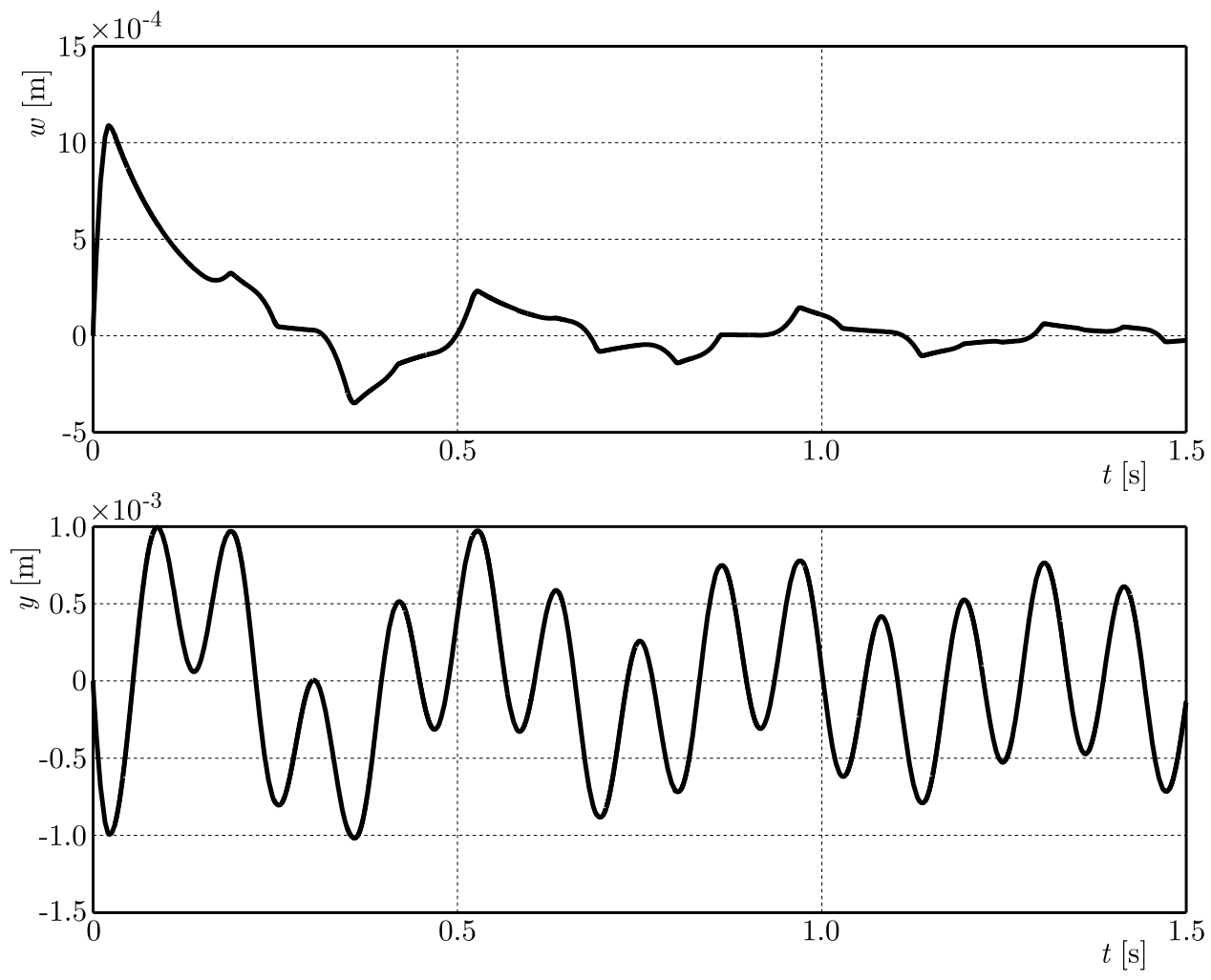

Fig. 13. Time histories of the engine point $C$ and frame displacements (algorithm ALG2, semi-active implementation) 
When comparing the simulation results for the proposed algorithms, the quantitative difference in motion of the subsytem to be vibroisolated can be observed. In the case of the ALG1, motion of the vibroisolated subsytem is typical vibration motion with a decreasing amplitude. This is similar to a great deal of such subsystem motion. The ALG2 is more effective and motion of the vibroisolated subsystem is characterized by a combination of both the oscillating and exponential motion.

\section{Summary}

This study investigates the potential application of a prototype MRSQD in a vehicle engine mount. Two algorithms for MRSQD control are proposed: one based on the inverse model of the engine-frame system (ALG1) and a sliding mode control algorithm (ALG2). In the ideal case, both algorithms ALG1 and ALG2 are effective, and the selected point of the engine can be returned to the position arbitrarily close to the static equilibrium position. In the case of semi-active implementation of ALG1 and ALG2, their effectiveness is significantly reduced. That applies particularly to ALG1, because semi-active actuators have a limited capability of reproducing the predetermined control force patterns. Despite this limitation, algorithms ALG1 and ALG2 can be the base for control of semi-active systems for vibration reduction.

Acknowledgement

This work has been supported by AGH University of Science and Technology under research program No. 11.11.130.958.

\section{References}

1. Flower W.C., 1997, Understanding hydraulic mounts for improved vehicle noise, vibration and ride qualities, SAE Paper, \#952666

2. Graf P.L., Shoureshi R., 1988, Modeling and implementation of semi-active hydraulic engine mounts, Journal of Dynamic Systems, Measurement Control, 110

3. Helber R., Doncker F., Bung R., 1990, Vibration attenuation by passive stiffness switching mounts, Journal of Sound and Vibration, 138, 1, 47-57

4. Imine H., Fridman L., Shraim H., Djemai M., 2011, Sliding Mode Based Analysis and Identification of Vehicle Dynamics, Springer Science and Business Media

5. Ivers D.E., Dol K., 1991, Semi-active suspension technology: An evolutionary view, ASME DE 40, Advanced Automotive Technologies, Book No. H00719, 1-18

6. Jędrzejowski J., 1986, Mechanics of crank mechanisms used in internal combustion engines (in Polish), WKŁ, Warszawa

7. Kamiński E., Pokorski J., 1983, Dynamics of vehicle suspensions and car transmission systems (in Polish), WKE, Warszawa

8. Kim J.H., 2014, Damping Force Control Filled with Magnetorheological Fluids and Engine Mount Having the Same, US Patent No. US 8,672,105 B2

9. SAPIŃSKi B., 2015, Theoretical analysis of magnetorheological damper characteristics in squeeze mode, Acta Mechanica et Automatica, 9, 2, 89-92

10. SAPIŃski B., GoŁDAsz J., 2015, Development and performance evaluation of an MR squeeze-mode damper, Smart Materials and Structures, 24, 115007

11. SapiŃski B., Krupa S., 2013, Vibration isolator with MR fluid in squeeze mode, Notification of Inventive Design, No. P.406179 
12. Shtessel Y., Edwards C., Fridman L., Levant A., 2014, Sliding Mode Control and Observation, Birkhäuser

13. Singh R., Kim G., Ravindra P.V., 1992, Linear analysis of automotive hydro-mechanical mount with emphasis on decoupler characteristics, Journal of Sound and Vibration, 158, 2, 219-243

14. Snamina J., Sapiński B., 2014, Analysis of an automotive vehicle engine mount based on squeezemode MR damper, Technical Transactions - Mechanics, 2-M/2014, 53-63

15. Utkin V.I., Chang H.C., 2002 Sliding mode control on electromechanical systems, Mathematical Problems in Engineering, 8, 451-473

16. Yu Y., Naganathan N.G., Dukkipati R.V., 2001, A literature review of automotive vehicle engine mounting systems, Mechanism and Machine Theory, 36, 123-142

Manuscript received October 28, 2015; accepted for print October 10, 2016 